\title{
Rapport de cas : Neuropathie optique ischémique postérieure
}

\author{
Hyukjin Kwon, OD \\ You \& Eye Optical Inc. \\ Sarah MacIver, OD, FAAO \\ École d'optométrie et des \\ sciences de la vision \\ Université de Waterloo
}

\section{Résumé}

\section{CONTEXTE}

La neuropathie optique ischémique postérieure (NOIP) est un trouble vasculaire qui survient dans la partie postérieure du nerf optique et qui, par conséquent, ne se présente habituellement pas avec un œedème du disque optique.

Rapport de cas : Nous rapportons ici un cas de NOIP avec une présentation non caractéristique. Ce rapport de cas souligne l'importance de comprendre les différentes physiopathologies de la NOIP et de la neuropathie optique ischémique antérieure (NOIA) pour aider à différencier les deux sur le plan clinique, et comprend une recension des écrits sur la NOIP.

\section{MOTS CLÉS :}

Neuropathie optique ischémique postérieure (NOIP), plexus vasculaire de la pie-mère, artères ciliaires postérieures (ACP), zone de partage de drainage

\section{INTRODUCTION}

Les neuropathies optiques ischémiques sont souvent différenciées en fonction de l'emplacement de l'ischémie et de l'étiologie. La neuropathie optique ischémique antérieure (NOIA) affecte le millimètre antérieur de la papille optique et se présente souvent avec un œè̀me du disque optique, ce qui entraîne une enflure visible du nerf optique. La NOIA peut être classée en fonction de son étiologie, non artéritique (NOIA-NA) ou artéritique (NOIA-A), qui est presque toujours associée à une artérite temporale. La neuropathie optique ischémique postérieure (NOIP) est une ischémie qui se présente à n'importe quel endroit derrière le disque optique et qui, par définition, ne cause pas l'enflure du nerf optique ${ }^{1,2}$. La NOIP est un diagnostic d'exclusion et est plus rare que la NOIA. La NOIA et la NOIP partagent certaines caractéristiques, y compris des facteurs de risque systémiques et une perte de vision soudaine et sans douleur. Nous pouvons établir une distinction clinique entre la NOIA et la NOIP si nous comprenons bien la physiopathologie de ces maladies. À l'heure actuelle, différentes options de traitement sont à l'étude et les corticostéroïdes systémiques semblent donner des résultats prometteurs pour les deux affections $s^{3,4}$.

Nous présentons ici un cas qui souligne l'importance de comprendre la pathogenèse et la présentation clinique de la NOIP pour la différencier de la NOIA. Il est important pour le fournisseur de soins oculovisuels primaires de comprendre les différents types de neuropathies optiques ischémiques afin de fournir un traitement rapide et approprié aux patients. 


\section{RAPPORT DE CAS}

Une femme sud-asiatique de 60 ans s'est présentée pour un examen oculaire d'urgence. La principale plainte de la patiente était l'apparition d'une douleur au cou suivie d'une perte soudaine de la vision. La patiente a signalé avoir commencé à ressentir une douleur diffuse au côté gauche du cou environ une semaine auparavant, suivie de la perte de vision trois jours auparavant. À l'examen, elle a signalé une détérioration de la vision, une douleur légère à l'œil (3 sur 10), des maux de tête au niveau du front et une photophobie. La patiente a affirmé ne pas éprouver les symptômes suivants : rougeur, écoulement, claudication de la mâchoire, sensibilité au cuir chevelu, fièvre, malaise ou antécédents de traumatisme oculaire récents ou de chirurgie. Elle a dit ne pas avoir d'éblouissements et ne pas voir de corps flottants. Ses antécédents médicaux étaient positifs pour l'hypothyroïdie et ses médicaments comprenaient le Synthroid.

Au moment de l'examen, son acuité visuelle corrigée à distance était de $6 / 6^{+2} \mathrm{OD}$ et $6 / 2 \mathrm{1}^{-2} \mathrm{OS}$. L'acuité au trou sténopéique à distance était de $6 / 18 \mathrm{OS}$. Le test $\mathrm{H}$ de Broad n'a révélé aucune restriction, aucune douleur lors des mouvements oculaires et aucune diplopie dans aucune direction du regard. Les résultats du test d'Amsler étaient normaux à l'OD; un scotome était visible dans le quadrant nasal inférieur de l'OS. Le test du bouchon rouge a révélé une saturation de $75 \%$ du côté de l'OS comparativement à une saturation de $100 \%$ du côté de l'OD. Le test de couleur d'Ishihara était normal à l'OD, mais seule la première planche était vue avec l'OS. Les deux pupilles étaient rondes et réactives à la lumière, avec un DPAR de grade 1 dans l'OS. L'évaluation du segment antérieur à la lampe à fente n'a rien révélé d'anormal. Les angles de Van Herick mesuraient 1:0,25. Les pressions intraoculaires étaient de $12 \mathrm{~mm} \mathrm{Hg}$ OU par tonométrie d'applanation de Goldmann. La gonioscopie avec verre à quatre miroirs a permis de voir le corps ciliaire dans le quadrant inférieur et l'éperon scléral dans les autres quadrants OU. L'examen du fond de l'œil dilaté a montré une cataracte corticale de grade $1 \mathrm{OU}$ avec trace de cataracte sous-capsulaire postérieure OS. L'examen du fond de l'œil (figures 1,2) par biomicroscopie du fond de l'œil et ophtalmoscopie indirecte binoculaire a révélé un vitré clair et un nerf rose et distinct avec un rapport excavation-papille de 0,65 OU. Les nerfs étaient gros et le tissu de l'anneau était intact dans les deux yeux. Le nerf optique ne montrait aucun œedème ou pâleur du disque, et aucun signe de défauts de la CFNR n'a été observé. Les résultats sur le nerf optique correspondaient à ceux mesurés par TCO-SD (Fig. 3). La macula était plate et la densité du pigment maculaire régulière; la vasculature était saine, avec un rapport artère-veine de $2 / 3 \mathrm{OU}$. La rétine périphérique ne présentait pas de trous, déchirures, détachement rétinien ou d'autre pathologie OU. L'analyse des cellules ganglionnaires (Fig. 4) et les examens à trame HD de la macula (Figs. 5,6) n’ont révélé aucune perte de cellules ganglionnaires et aucune anomalie de la macula OU. Un examen du champ visuel Humphrey 30-2 (Figs. 7,8) a été effectué avant la dilatation. L'œil droit n'avait rien de particulier, avec des défauts non spécifiques en supérieur partout dans le champ de vision. L'examen de l'œil gauche a révélé un défaut altitudinal profond du champ visuel en inférieur et un défaut arciforme en supérieur. Le défaut observé en inférieur était plus profond que le défaut en supérieur. L’anomalie du champ visuel dans l'œil gauche ne correspondait pas aux résultats cliniques pour le nerf optique, la rétine ou la $\mathrm{TCO}$, ce qui suggère une pathologie postérieure à la lamina.

Un renvoi urgent a été fait au médecin de famille pour les analyses sanguines, y compris la vitesse de sédimentation (VS), la protéine C-réactive (PCR) et une demande de consultation a été faite à l'ophtalmologiste de garde pour exclure une neuropathie optique ischémique postérieure secondaire à une artérite temporale. On a demandé une IRM de l'orbite et du chiasme ainsi qu'une IRM du cou afin d'exclure toute lésion plus postérieure ou la possibilité d'une dissection carotidienne. La douleur oculaire très faible devait être réévaluée une fois la cause de la perte de vision diagnostiquée.

Les résultats des analyses sanguines ont montré une VS, une PCR et des plaquettes normales. L'ophtalmologiste de garde a vu la patiente le lendemain. Les résultats de l'examen étaient semblables à ceux de la veille, mais l'acuité visuelle OS de la patiente s'était détériorée à 20/100. Malgré l'absence de signes et de symptômes classiques, l'ophtalmologiste a d'abord diagnostiqué une NOIA-NA chez la patiente. Aucun traitement n'a été entrepris au moment de l'investigation et la vision s'est lentement améliorée au cours des 12 mois suivants. Lors du suivi à un an, on n'a noté aucune pâleur de la papille et aucune perte de la CFNR. L’anomalie du champ visuel était toujours présente, mais s'était améliorée. D'autres réflexions sur le cas indiquaient la possibilité d'un diagnostic de NOIP en raison du manque de signes cliniques. Les résultats de l'IRM n'avaient rien de particulier. 
Figure 1 : La photographie rétinienne de l'OD au moment de l'examen initial révèle l'absence d'œè̀me discal, d'hémorragie et de pâleur du nerf optique, une densité uniforme de pigment maculaire et une vasculature saine.

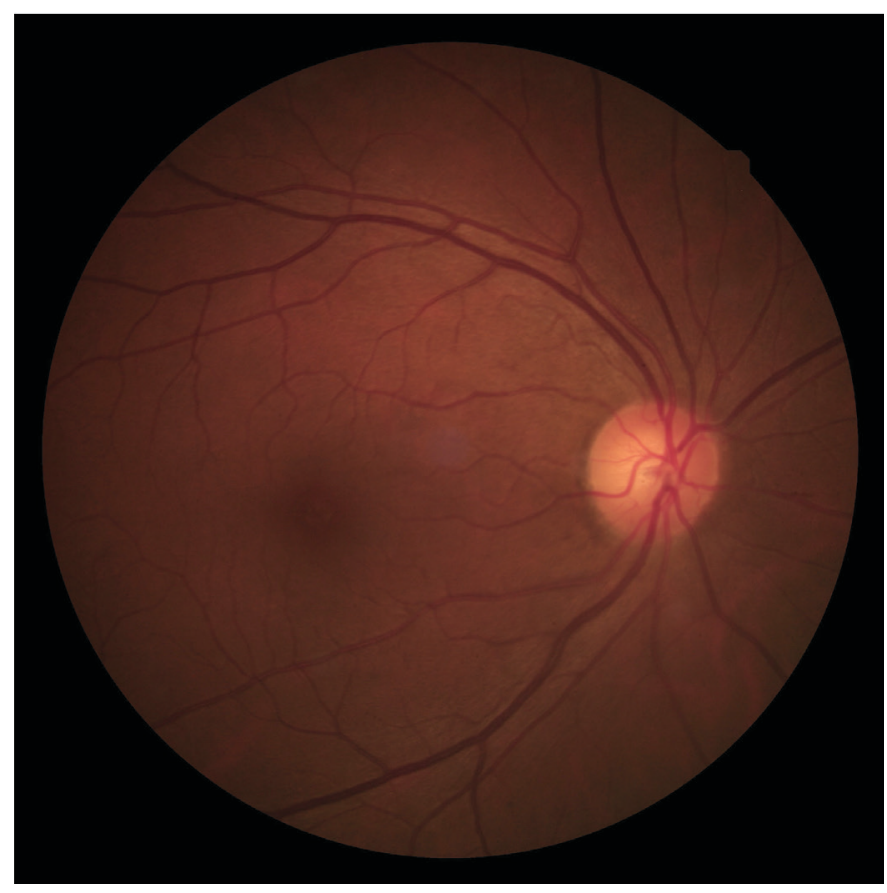

Figure 2: La photographie rétinienne de l'OS au moment de l'examen initial révèle l'absence d'œè̀me discal, d'hémorragie et de pâleur du nerf optique, une densité uniforme de pigment maculaire et une vasculature saine.

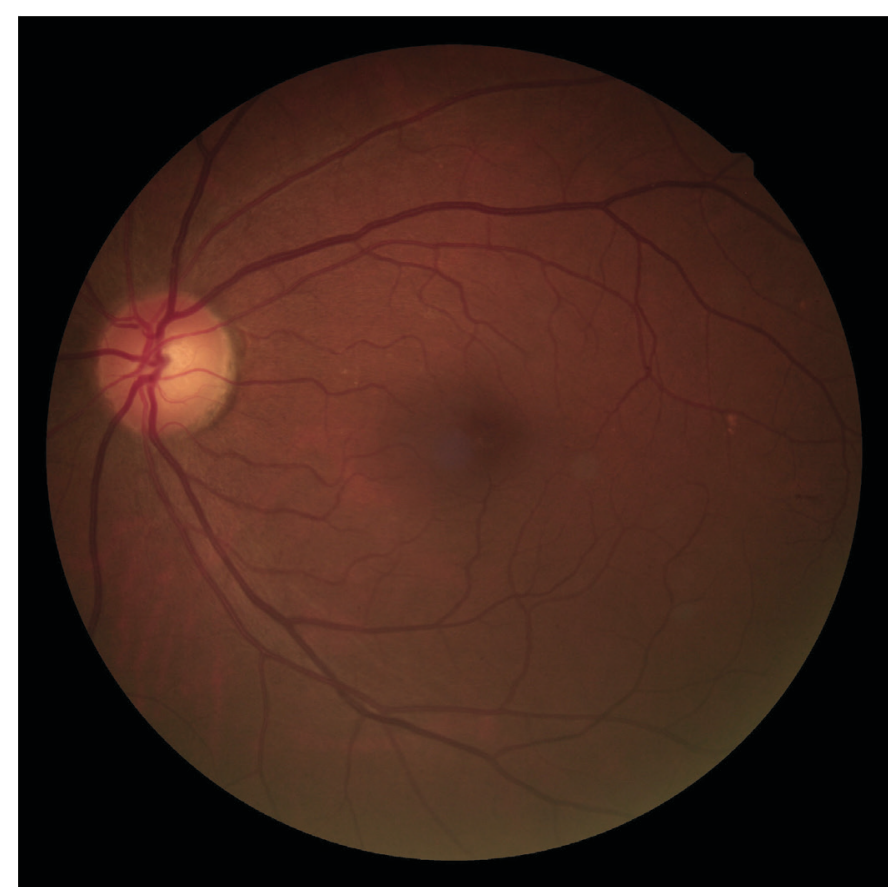


Figure 3 : À l'examen initial, la TCO (Zeiss Cirrus HD-OCT) de la papille et de la CFNR OU montrent une CFNR saine sans signe d'oedème de la papille $O U$.
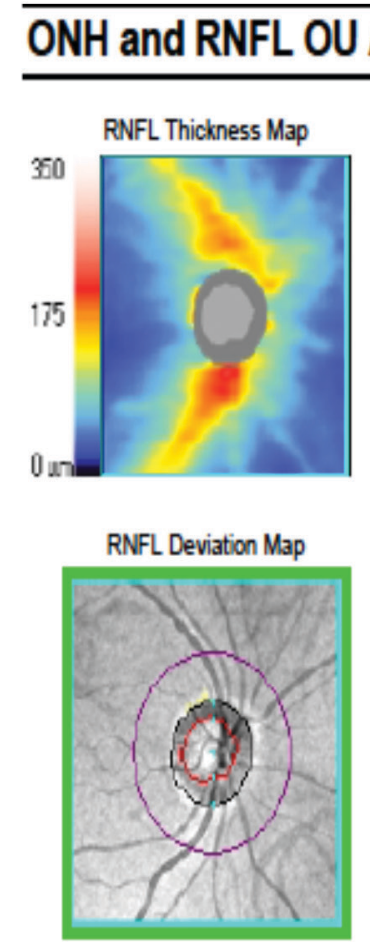

Disc Center $(0.12,0.03) \mathrm{mm}$

Extracted Horizontal Tomogram

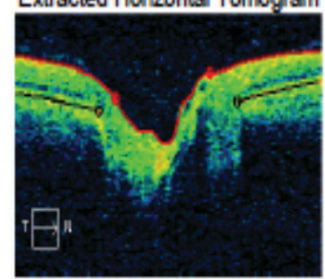

Extracted Vertical Tomogram

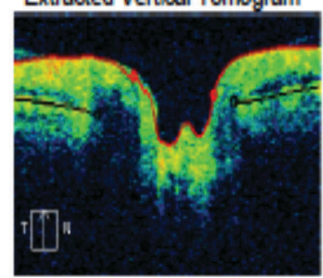

RNFL Circular Tomogram

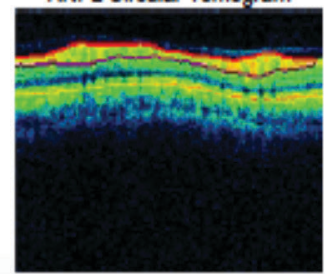

\begin{tabular}{|c|c|c|}
\hline$\Delta$ & OD & os \\
\hline Averads RNFL Thisloness & $97 \mu m$ & $103 \mu \mathrm{n}$ \\
\hline FuFL Syminerry & \multicolumn{2}{|c|}{$94 \%$} \\
\hline Rin Areo & $1.42 \mathrm{~mm}^{2}$ & $1.48 \mathrm{~mm}^{2}$ \\
\hline Disc Ates & $247 \mathrm{~mm}^{2}$ & $2.63 \mathrm{~mm}^{2}$ \\
\hline Average C.D Rotio & 0.64 & 0.65 \\
\hline Yelical C.D Rिtio & 060 & 0.60 \\
\hline Cup Volute & $0.251 \mathrm{~mm}^{3}$ & $0.255 \mathrm{~mm}^{3}$ \\
\hline
\end{tabular}

Neuro-retinal Rim Thickness

$\mu \mathrm{n}-\mathrm{OD} \ldots \mathrm{DS}$

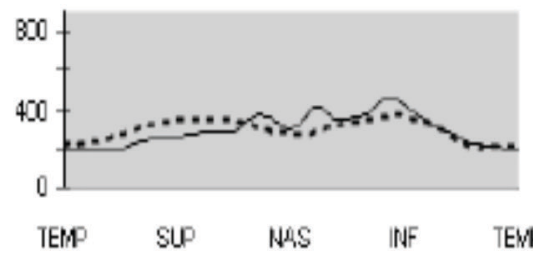

RNFL Thickness
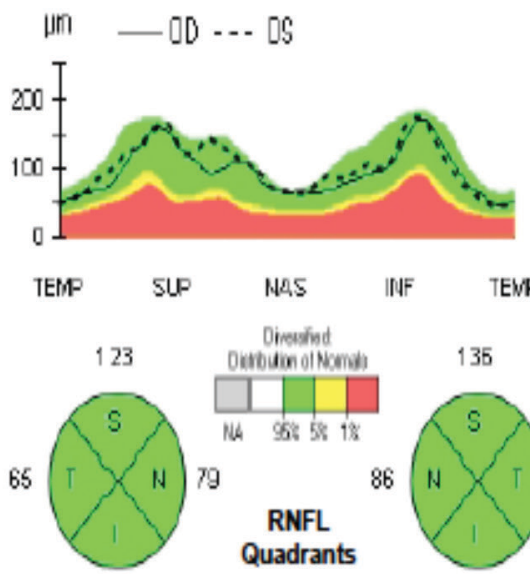

121

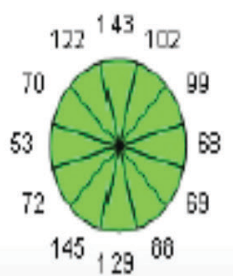

14512900
RNFL

Clock

Hours

RNFL
Quadrants

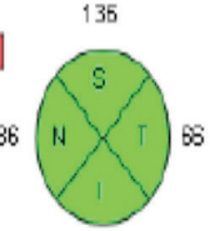

124

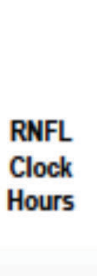

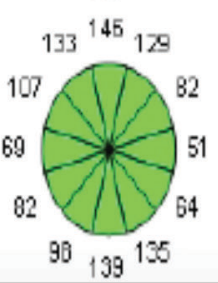

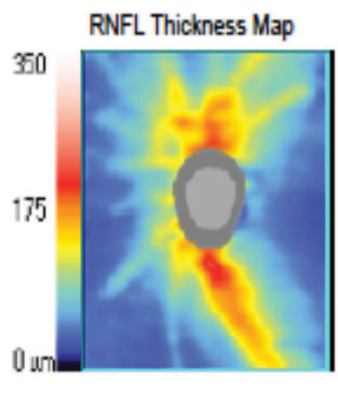

RNFL Deviation Map

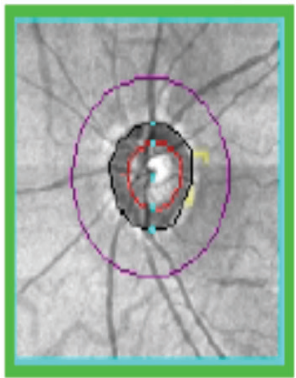

Disc Center $(0.03,0.27) \mathrm{mm}$ Extracted Horizontal Tomogram

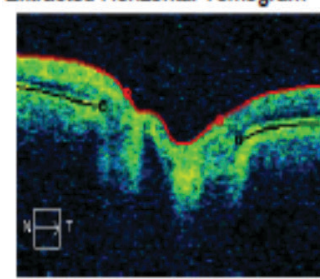

Extracted Vertical Tomogram

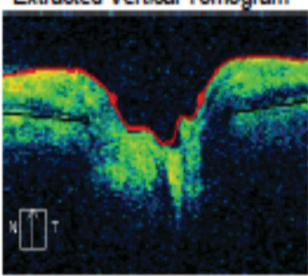

RNFL Circular Tomogram

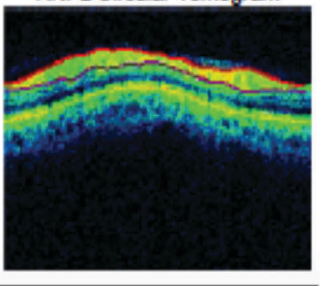


Figure 4 : L'examen Cube maculaire (Zeiss Cirrus HD-OCT, analyse de la couche de cellules ganglionnaires) OU à l'examen initial montre une couche de CG saine dans les deux yeux. Il n'y a pas de détérioration hémisphérique pouvant être corrélée au défaut du champ visuel de l'œil droit.

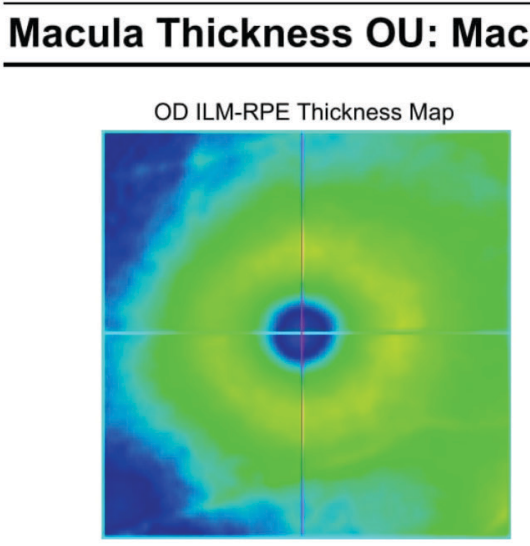

Fovea: 251,64
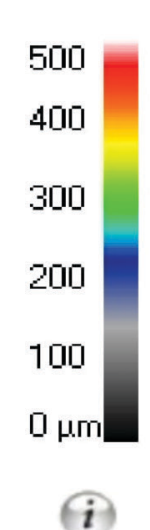

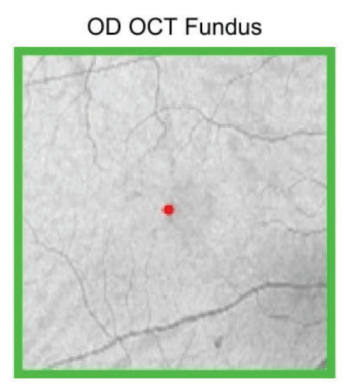

OD ILM-RPE Thickness
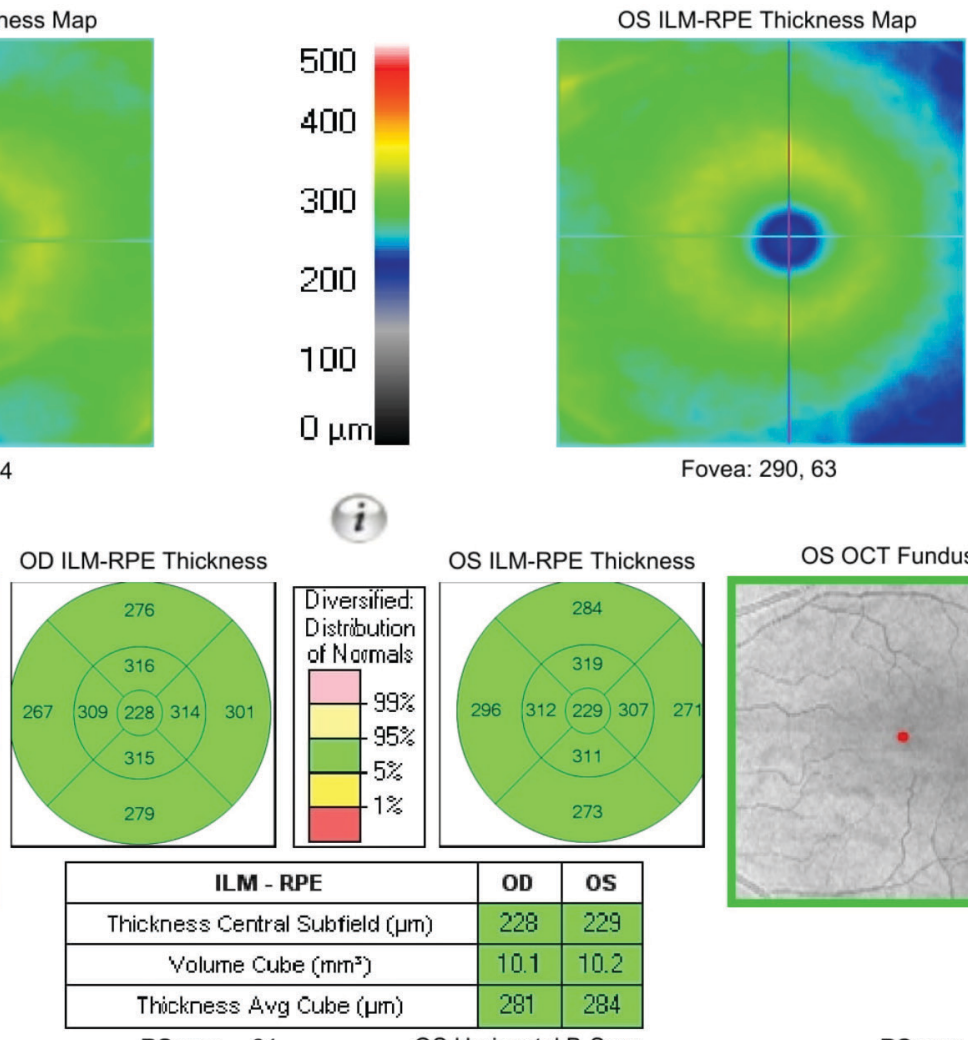

Fovea: 290, 63

\section{OD O OS}

\begin{tabular}{|c|c|c|}
\hline ILM - RPE & OD & os \\
\hline Thickness Central Subtield ( $\mu \mathrm{r})$ & 228 & 229 \\
\hline Volume Cube $\left(\mathrm{m} \mathrm{m}^{3}\right)$ & 10.1 & 10.2 \\
\hline Thickness Avg Cube (um) & 281 & 284 \\
\hline
\end{tabular}
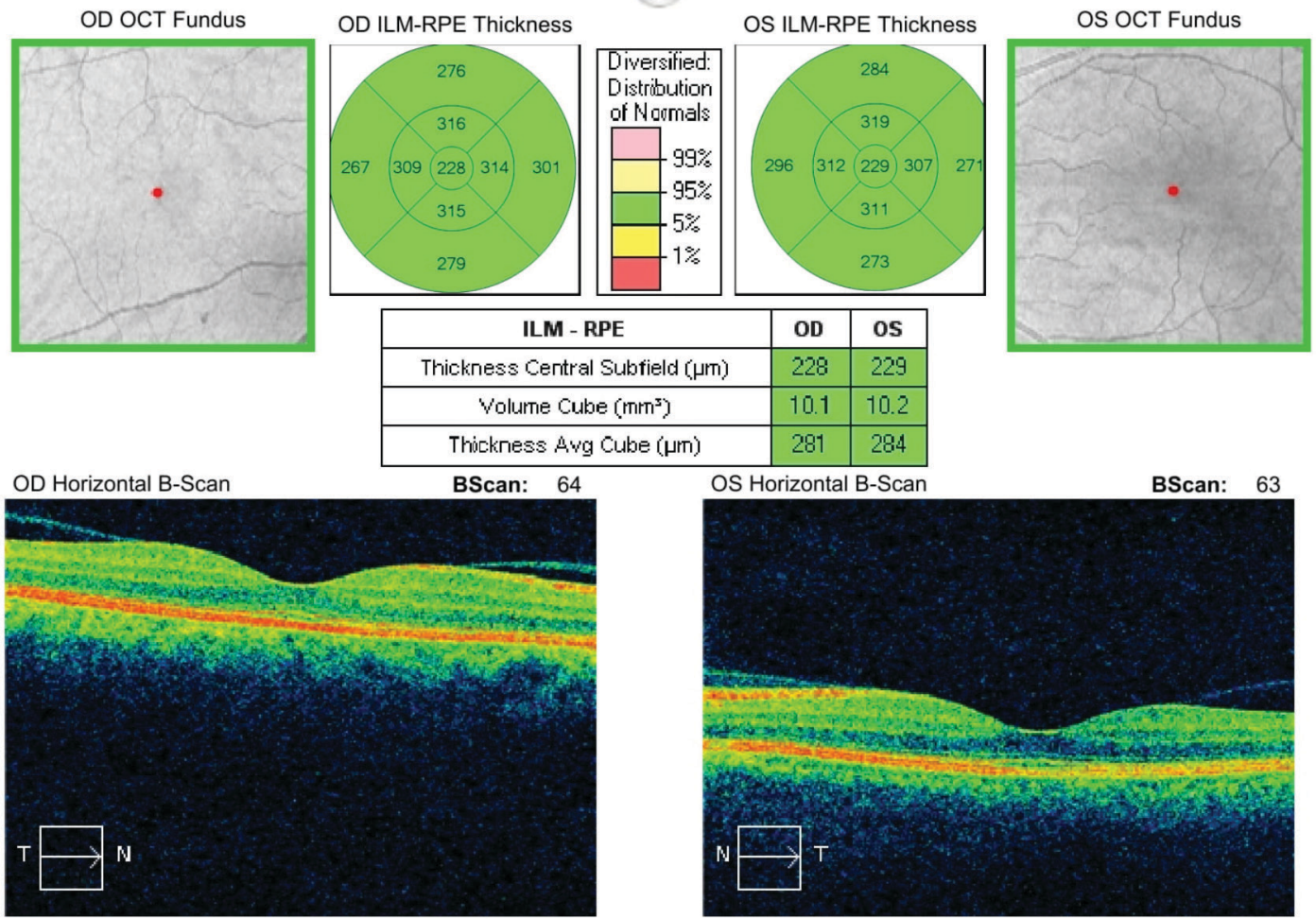
Figure 5 : Au moment de l'examen initial, l'examen à trame de 5 lignes HD (Zeiss Cirrus HD-OCT) OD montre une macula saine et l'absence de pathologie pouvant être à l'origine de la détérioration de la vision.

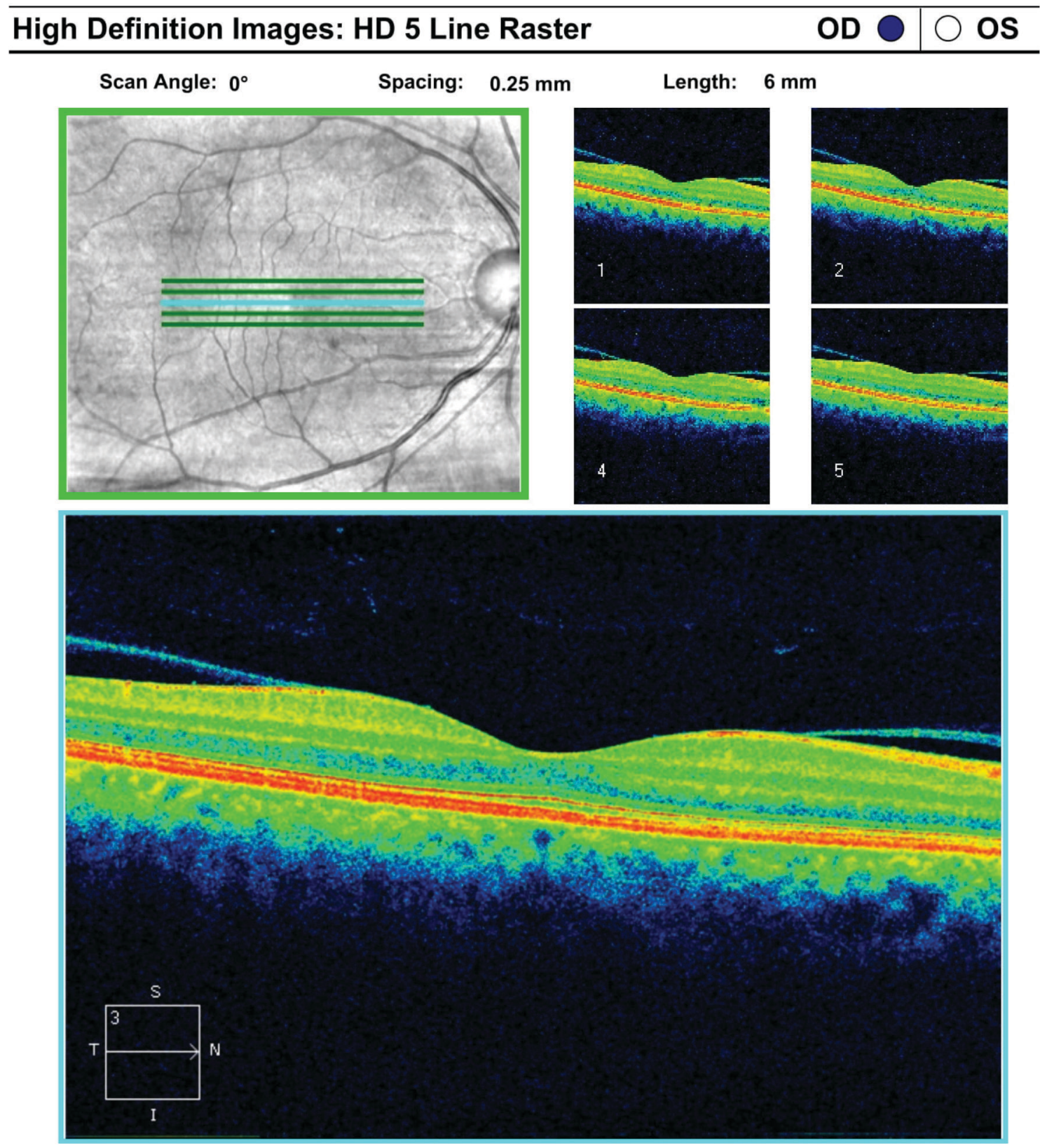


Figure 6 : L'examen à trame de 5 lignes HD (Zeiss Cirrus HD-OCT) OS à l'examen initial montre une macula saine.

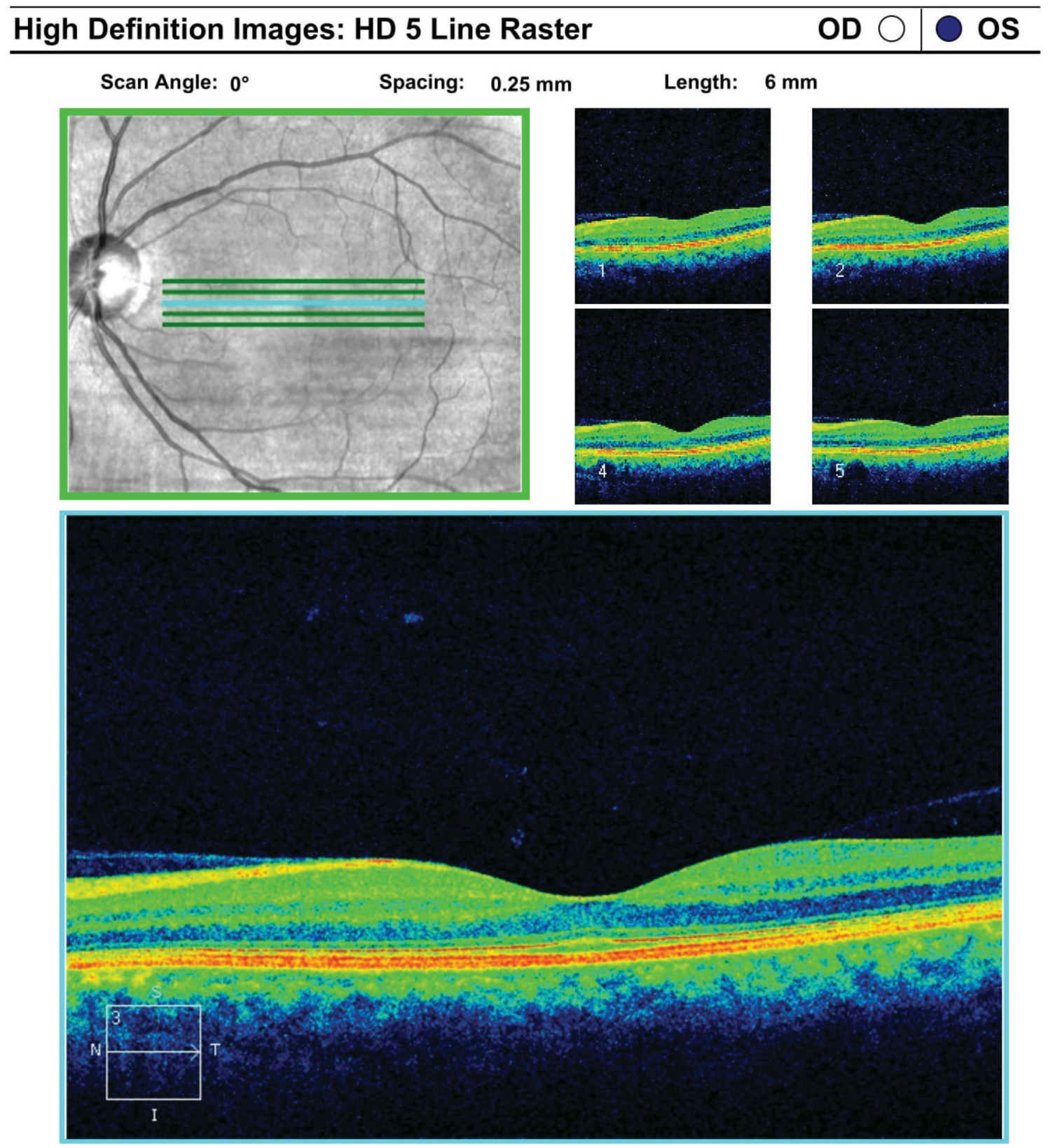


Figure 7 : Le champ visuel Humphrey 30-2 SITA Standard OD au suivi montre des défauts légers et éparpillés dans la région supérieure.

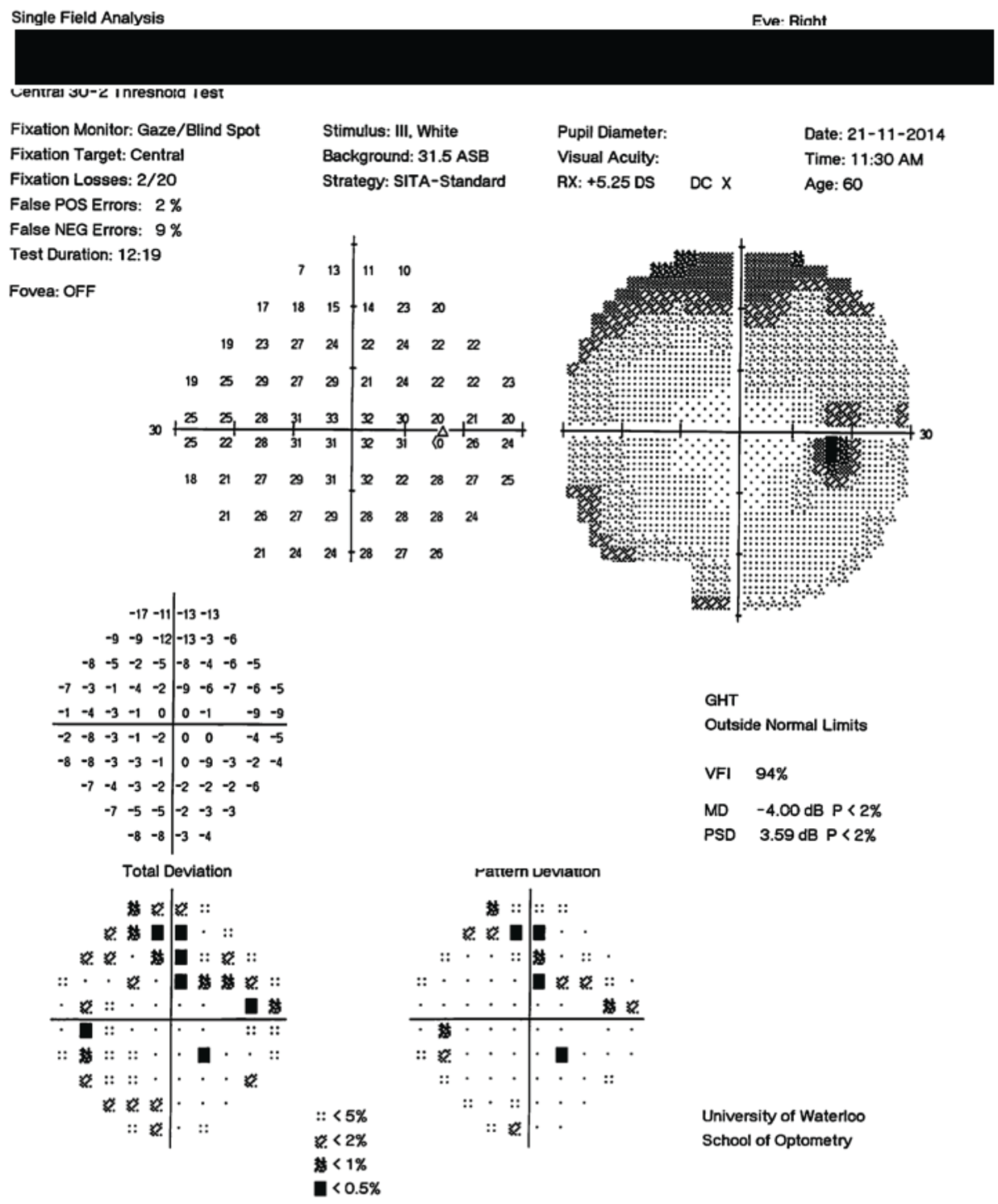

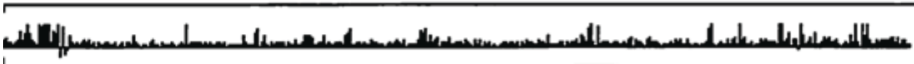

- 2010 Carl Zeiss Meditec HFA II 740-40962-5.1.2/5.1.2 
Figure 8 : L'examen du champ visuel Humphrey 30-2 SITA Standard OS au suivi montre un défaut altitudinal profond en inférieur et un défaut arciforme en supérieur.

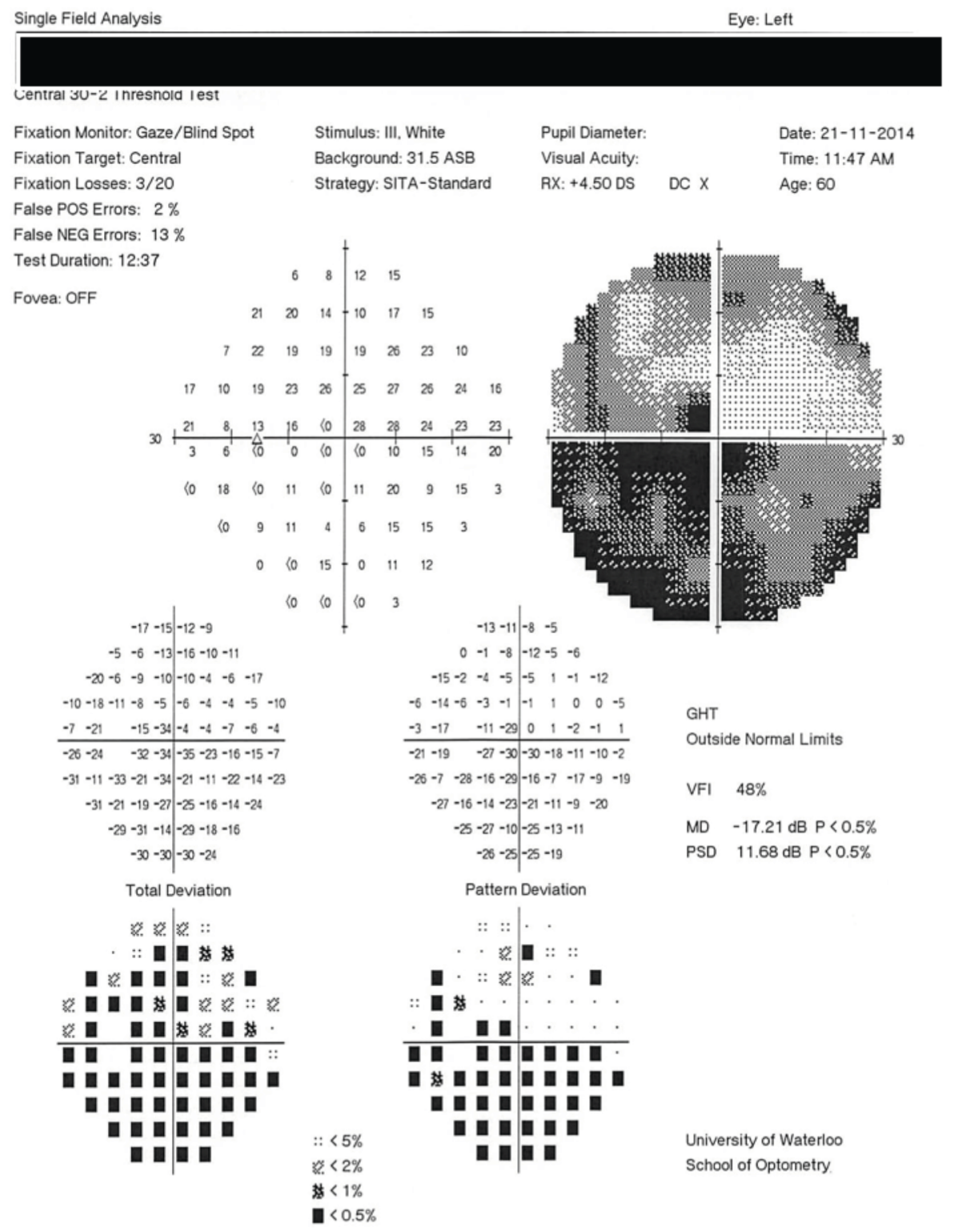

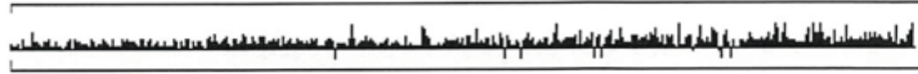

- 2010 Carl Zeiss Meditec

HFA II 740-40962-5.1.2/5.1.2 
DISCUSSION

Les neuropathies optiques ischémiques peuvent être différenciées sur la base de l'emplacement de l'ischémie. L'ischémie se trouve le plus souvent dans la lame antérieure et cause des signes cliniques classiques au niveau du nerf optique et de la vision. Lorsque l'ischémie est derrière la lame, il y a une perturbation visuelle, mais le nerf optique n'est pas affecté. La NOIA et la NOIP sont semblables en ce sens qu'elles peuvent être classées dans des étiologies artéritiques et non artéritiques.

Pour mieux comprendre la NOIP, il est utile de revoir la pathologie de la NOIA. La NOIA est généralement classée comme artéritique (NOIA-A) ou non artéritique (NOIA-NA). On peut différencier la NOIA-A de la NOIA-NA selon qu'il s'agit d'une pathogenèse secondaire à une vasculite ou à d'autres conditions ${ }^{5}$. La NOIA-A est le plus souvent causée par l'artérite temporale, un type particulier de vasculite. Des analyses sanguines, y compris la VS et la PCR, ainsi qu'un historique de cas complet sont nécessaires pour enquêter sur la présence d'inflammation si l'on soupçonne la présence d'une NOIA-A. Les symptômes, y compris les maux de tête, la sensibilité du cuir chevelu de même que la sensibilité au niveau de l'artère touchée et la claudication de la mâchoire, sont souvent présents chez les patients atteints d'artérite temporale. De plus, on observe fréquemment une myalgie, de la fatigue, une perte de poids et une diminution de l'appétit. Si l'on soupçonne une artérite temporale, un traitement immédiat est requis ${ }^{1,6}$. L'artérite temporale est une véritable urgence oculaire et une perte de vision permanente s'ensuivra si elle n'est pas prise en charge le plus rapidement possible.

La pathogenèse de la NOIA-NA n'est toujours pas claire, mais semble englober les sources courantes de lésions ischémiques, y compris, mais sans s'y limiter, la thromboembolie, l'hypotension systémique et l'occlusion athérosclérotique $^{5}$. Comme la NOIA-NA n'est pas attribuable à une inflammation, les marqueurs inflammatoires ne seraient pas élevés dans un test de laboratoire. Dans le cas décrit ici, la VS, la PCR et les plaquettes étaient normales, ce qui excluait la NOIA-A. De plus, la présentation du champ visuel correspondait davantage à la NOIA-NA qu'à la NOIA-A. La patiente s'est présentée avec un défaut altitudinal unilatéral du champ visuel en inférieur, une présentation fréquente dans les cas de NOIA$\mathrm{NA}^{7}$. Les études montrent que la TCO est utile pour le diagnostic de la NOIA-NA ${ }^{8}$. Après un gonflement axonal aigu puis un amincissement, la CCG et la CFNR montreraient toutes deux un épaississement puis un amincissement des couches ${ }^{8}$. De plus, l'emplacement et la gravité du défaut du champ visuel sont en corrélation significative avec l'amincissement de la CFNR ${ }^{8}$. Par conséquent, la NOIA-A et la NOIA-NA devraient être accompagnées d'un œedème du disque optique et d'un épaississement (stade aigu) ou d'un amincissement (après résolution de l'œè̀me) de la CFNR et de la CCG par TCO, ce qui n'a pas été observé dans ce cas $^{8,9}$. De même, une pathologie de la rétine ou d'un autre nerf optique peut également être exclue comme cause du défaut du champ visuel puisque la TCO de la macula et du nerf optique était normale ${ }^{10,11}$. La couche de fibres nerveuses a montré une différence de 31 microns en supérieur du côté nasal, ce qui suggère une légère asymétrie entre l'œil droit et l'œil gauche. L'asymétrie de la CFNR dans le quadrant nasal supérieur et l'absence d'amincissement hémisphérique observée lors de l'analyse des cellules ganglionnaires de l'œil gauche n'expliquent pas le scotome diffus et profond dans le champ visuel $\mathrm{OS}^{8,12}$. En se basant sur l'âge de la patiente, ses symptômes, ses résultats cliniques et ses résultats de laboratoire, on peut encore supposer sans risque qu'il s'agissait d'un événement ischémique non artéritique, mais la localisation de l'ischémie était probablement postérieure, ce qui suggère un diagnostic de NOIP.

Les NOIP peuvent être classées en trois types différents : artéritiques, non artéritiques et périopératoires ${ }^{13}$. L'épidémiologie exacte ou la prévalence des NOIP est inconnue, mais dans une étude de Hayreh, l'âge médian des patients souffrant d'une NOIP non artéritique était de 61,5 ans et celui des patients souffrant d'une NOIP artéritique était de 73,5 ans $^{13}$. Il existe un chevauchement considérable avec les NOIA en termes de symptômes, d'association systémique, de tests diagnostiques et de protocole de traitement. Comme dans le cas de la NOIA, certains des facteurs de risque comprennent l'anémie, l'hypotension intraopératoire, le diabète, l'obésité et la perte de sang intraopératoire, qui peuvent diminuer la pression de perfusion oculaire ${ }^{14-16}$.

Pour mieux comprendre pourquoi la NOIP est un diagnostic par exclusion, il est utile d'examiner le système vasculaire du nerf optique. La papille est alimentée par l'artère rétinienne centrale (ARC) et les artères ciliaires postérieures courtes (ACPC) ${ }^{17}$. La partie postérieure du nerf est alimentée par l'ARC dans les plexus vasculaires central et pial, qui sont responsables du système vasculaire périphérique ${ }^{13}$. Dans la NOIA, l'ischémie est due aux ACPC ${ }^{3}$. Les ACPC sont le point d'aboutissement du système artériel, sans anastomoses aux autres branches artérielles ${ }^{13}$. Aucun autre vaisseau sanguin ne peut compenser les ACPC si elles deviennent ischémiques ${ }^{13}$. Dans la partie postérieure du nerf optique, le plexus vasculaire pial provient de la choroïde péripapillaire, du cercle de Zinn et Haller, de l'artère rétinienne centrale et de l'artère ophtalmique ${ }^{13}$. Le plexus vasculaire pial n'est pas le point d'aboutissement du système artériel, le sang peut donc se déplacer librement dans le plexus collatéral. Cela sert de facteur de protection pour la partie postérieure du nerf si une région d'un système collatéral devient ischémique ${ }^{13}$. Par conséquent, il y a moins de risque d'ischémie dans la partie postérieure du nerf optique. 
Parmi les cas de NOIP-NA décrits par Hayreh, 24 des 35 yeux présentaient une acuité visuelle de 20/100 ou moins ${ }^{13}$. À l'examen initial, notre patiente présentait une acuité visuelle de 20/70. Cependant, l'évaluation effectuée le lendemain par un ophtalmologue a montré une nouvelle détérioration de l'acuité visuelle, qui a atteint 20/100 ${ }^{13}$. Dans l'étude de Hayreh, tous les patients ont subi un examen oculaire approfondi ainsi qu'une angiographie à la fluorescéine, une imagerie par résonance magnétique et une évaluation neurologique afin d'exclure tout autre trouble oculaire, du nerf optique ou neurologique ${ }^{13}$. Dans ce cas, avec une douleur oculaire légère (3/10) et l'absence d'œdème du disque optique, l'IRM a permis d'exclure une névrite optique rétrobulbaire secondaire à la sclérose en plaques. De plus, la névrite optique démyélinisante se produit le plus souvent chez les jeunes femmes de 20 à 45 ans s,19. $^{18}$.

Les défauts du champ visuel observés dans les neuropathies optiques ischémiques peuvent varier selon la partie de la CFNR qui est endommagée. Dans le cas de la NOIP, les études montrent que le défaut du champ visuel le plus courant est un défaut central, mais d'autres défauts peuvent être observés ${ }^{1,13}$. Le type de défaut du champ visuel est déterminé par le système vasculaire qui est affecté20 ${ }^{2}$. Dans la rétine, le faisceau papillomaculaire de la CFNR sort de l'aspect temporal et constitue le noyau central du nerf optique ${ }^{1}$. La partie postérieure du nerf optique n'est pas alimentée par l'ARC, mais seulement par les extrémités capillaires terminales du plexus vasculaire pial ${ }^{12}$. Ce plexus terminal est appelé la zone de partage de drainage ${ }^{1}$. Il a été démontré que la zone de partage de drainage est la région la plus vulnérable à l'ischémie ${ }^{1,21}$. Dans les cas de diminution de la perfusion, la zone de partage de drainage sera la première région à devenir ischémique en raison d'un manque d'apport sanguin au noyau central du nerf optique ${ }^{1}$. Il en résulte une anomalie du champ visuel central qui est le plus souvent présente chez les patients atteints de NOIP1. Comme le plexus vasculaire pial alimente différentes parties du nerf optique postérieur, une ischémie localisée peut présenter différents types de défauts du champ visuel, selon la zone du plexus qui est touchée et l'endroit où les fibres du nerf optique touchées prennent naissance dans la rétine ${ }^{13}$. En fait, Hayreh a indiqué que seuls 9 des 32 yeux de son étude présentaient des défauts centraux et que les autres présentaient divers défauts du champ visue ${ }^{13}$. Dans notre cas, la patiente n'avait pas de défaut central, mais présentait plutôt un défaut altitudinal en inférieur avec quelques scotomes du côté nasal. D'après la physiopathologie de la NOIP, il n'est pas impossible de déceler un défaut altitudinal en inférieur chez cette patiente, mais il peut s'agir d'une forme rare d'un défaut du champ visuel.

Le traitement de la NOIP-NA est semblable à celui de la NOIA-NA. Selon Hayreh, le traitement systémique par corticostéroïdes entraîne généralement des améliorations significatives de l'acuité visuelle et du champ visuel ${ }^{22-24}$. Dans une étude de Hayreh, une cohorte de 696 yeux consécutifs avec une NOIA-NA, vus dans les 2 semaines suivant son apparition, et une acuité visuelle initiale de 20/70 ou moins a été assignée au traitement systémique par corticostéroïdes ou au non-traitement ${ }^{22}$. Six mois après la visite initiale, $69,8 \%$ des patients du groupe traité par corticostéroïdes présentaient une amélioration de l'acuité visuelle, contre seulement $37,1 \%$ dans le groupe non traité2 ${ }^{22}$. Une comparaison similaire a été faite pour les améliorations des défauts du champ visuel; après 6 mois, $40,1 \%$ du groupe traité par corticostéroïdes systémiques ont montré une amélioration contre seulement $24,5 \%$ de ceux qui n'ont pas reçu de corticothérapie ${ }^{22}$. Dans ces études, le traitement par corticostéroïdes a été poursuivi jusqu'à la résolution complète de l'œè̀me du disque optique. Toutefois, comme la NOIP ne présente pas d'œedème du disque optique, les avantages du traitement par corticostéroïdes et sa durée sont toujours à l'étude.

Il n'y a pas de protocole de suivi défini pour la NOIP. Dans une étude, Hayreh a commencé un traitement par stéroïdes pendant 2 jours, suivi d'une réduction rapide et d'une évaluation de suivi en moins de 2 semaines pour la NOIP-NA et la NOIP périopératoire ${ }^{13}$. Cependant, le suivi à long terme après l'apparition initiale variait de 1,3 à 214 mois pour la NOIPNA, de 1,3 à 55,6 mois pour la NOIP-A et de 4,6 à 56,6 mois pour la NOIP périopératoire. Hayreh explique que le pronostic concernant le rétablissement visuel peut également varier selon le type de NOIP. Dans la NOIP-NA, la vision peut être améliorée par une thérapie à forte dose de corticostéroïdes, alors que le traitement de la NOIP-A ne fait qu'empêcher une perte de vision supplémentaire. Dans la NOIP-NA, la résolution spontanée de l'acuité visuelle et du champ peut se produire sans traitement. En fait, seulement 5 des 16 yeux traités sont demeurés stables, pour 9 des 16 yeux non traités ${ }^{13}$.

\section{CONCLUSION}

La neuropathie optique ischémique postérieure (NOIP) est un diagnostic d'exclusion qui peut être posé après avoir fait l'historique complet du patient, l'examen à la présentation et des tests diagnostiques appropriés. Ce cas montre l'importance d'une prise en charge en collaboration avec les ophtalmologistes et les médecins de famille dans tout cas de neuropathie optique ischémique présumée, y compris la présence présumée de NOIP. En tant que fournisseurs de soins oculovisuels primaires, les optométristes doivent connaître les différences entre les diverses neuropathies optiques et la façon de trouver une prise en charge appropriée et rapide pour leurs patients. $\bullet$ 


\section{RÉFÉRENCES}

1. Sadda SR, Nee M, Miller NR, Biousse V, Newman NJ, Kouzis A. Clinical spectrum of posterior ischemic optic neuropathy. Am J Ophthalmol 2001;132(5):743-50.

2. Behbehani R. Clinical approach to optic neuropathies. Clin Ophthalmol 2007;1(3):233-46

3. Atkins EJ, Bruce BB, Newman NJ, Biousse V. Treatment of nonarteritic anterior ischemic optic neuropathy. Surv Ophthalmol 2010;55(1):47-63.

4. Hayreh SS. Management of ischemic optic neuropathies. Indian J Ophthalmol 2011;59(2):123-36.

5. Patel HR, Margo CE. Pathology of ischemic optic neuropathy. Arch Pathol Lab Med 2017;141(1):162-6. doi: 10.5858/arpa.20160027-RS [doi].

6. Chacko JG, Chacko JA, Salter MW. Review of giant cell arteritis. Saudi J Ophthalmol 2015;29(1):48-52.

7. Han S, Jung JJ, Kim US. Differences between non-arteritic anterior ischemic optic neuropathy and open angle glaucoma with altitudinal visual field defect. Korean J Ophthalmol 2015;29(6):418-23. doi: 10.3341/kjo.2015.29.6.418 [doi].

8. Alasil T, Tan O, Lu AT, et al. Correlation of Fourier domain optical coherence tomography retinal nerve fiber layer maps with visual fields in nonarteritic ischemic optic neuropathy. Ophthalmic Surg Lasers Imaging 2008;39:S71-9.

9. Bellusci C, Savini G, Carbonelli M, Carelli V, Sadun AA, Barboni P. Retinal nerve fiber layer thickness in nonarteritic anterior ischemic optic neuropathy: OCT characterization of the acute and resolving phases. Graefes Arch Clin Exp Ophthalmol 2008;246(5):641-7.

10. Kim EK, Park HL, Park CK. Relationship between retinal inner nuclear layer thickness and severity of visual field loss in glaucoma. Sci Rep 2017;7(1):5543.

11. Suh W, Lee JM, Kee C. Depth and area of retinal nerve fiber layer damage and visual field correlation analysis. Korean J Ophthalmol 2014; 28(4):323-9.
12. Zhang X, Rasa AS, De Moraes GV, Hood DC. Mapping the visual field to retinal ganglion cell thickness image. Invest Ophthalmol Vis Sci 2011; 52:5085.

13. Hayreh SS. Posterior ischaemic optic neuropathy: Clinical features, pathogenesis, and management. Eye (Lond) 2004;18(11):1188-206.

14. Nickels TJ, Manlapaz MR, Farag E. Perioperative visual loss after spine surgery. World J Orthop 2014;5(2):100-6.

15. Epstein NE. Perioperative visual loss following prone spinal surgery: A review. Surg Neurol Int 2016;7(Suppl 13):347.

16. Cheng MA, Todorov A, Tempelhoff R, McHugh T, Crowder CM, Lauryssen C. The effect of prone positioning on intraocular pressure in anesthetized patients. Anesthesiology 2001;95(6):1351-5.

17. Cioffi GA. Ischemic model of optic nerve injury. Trans Am Ophthalmol Soc 2005;103:592-613.

18. Dooley, MC, Foroozan R. Optic neuritis. J Ophthalmic Vis Res. 2010;5(3):182-7.

19. Dworak DP, Nichols J. A review of optic neuropathies. Dis Mon 2014;60(6):276-81.

20. Hayreh SS, Zimmerman MB. Optic disc edema in non-arteritic anterior ischemic optic neuropathy. Graefes Arch Clin Exp Ophthalmol 2007;245(8):1107-21.

21. Hayreh SS. Posterior ciliary artery circulation in health and disease: the Weisenfeld lecture. Invest Ophthalmol Vis Sci 2004;45(3):74957. doi: 10.1167/iovs.03-0469 [doi].

22. Hayreh SS, Zimmerman MB. Non-arteritic anterior ischemic optic neuropathy: Role of systemic corticosteroid therapy. Graefes Arch Clin Exp Ophthalmol 2008;246(7):1029-46.

23. Hayreh SS, Zimmerman B, Kardon RH. Visual improvement with corticosteroid therapy in giant cell arteritis. report of a large study and review of literature. Acta Ophthalmol Scand 2002;80(4):355-67.

24. Hayreh SS, Zimmerman B. Visual deterioration in giant cell arteritis patients while on high doses of corticosteroid therapy. Ophthalmology 2003;110(6):1204-15. 